\title{
In vivo Imaging Biodistribution Profile of a New Macrocyclic Gadolinium Chelate as a Highly Stable Multifunctional MRI Contrast Agent
}

\author{
Bo Kyung Sung ${ }^{1}$, Yeong Woo $\mathrm{Jo}^{2}$, Yongmin Chang ${ }^{1,3,4}$ \\ ${ }^{1}$ Department of Medical \& Biological Engineering, Kyungpook National University, Daegu, Korea \\ ${ }^{2}$ Hana Pharm Co., Pangyo R\&D Center, Seongnam-si, Korea \\ ${ }^{3}$ Department of Molecular Medicine, School of Medicine, Kyungpook National University, Daegu, \\ Korea \\ ${ }^{4}$ Department of Radiology, Kyungpook National University Hospital, Daegu, Korea
}

\section{Technical Note}

Received: October 23, 2018

Revised: November 13, 2018

Accepted: November 19, 2018

\section{Correspondence to:}

Yongmin Chang, Ph.D. Department of Molecular Medicine \& Radiology, School of Medicine, Kyungpook National University, 200, Dongduk-ro, Jung-gu, Daegu 700-714, Korea. Tel. +82-53-420-5471

Fax. +82-53-422-2677

E-mail: ychang@knu.ac.kr

This is an Open Access article distributed under the terms of the Creative Commons Attribution Non-Commercial License (http://creativecommons.org/licenses/ by-nc/4.0/) which permits unrestricted non-commercial use, distribution, and reproduction in any medium, provided the original work is properly cited.

Copyright (C) 2019 Korean Society of Magnetic Resonance in Medicine (KSMRM)
Gadolinium contrast agents (CAs) are integral components of clinical magnetic resonance imaging (MRI). However, safety concerns have arisen regarding the use of gadolinium CAs, due to their association with nephrogenic systemic fibrosis (NSF). Furthermore, recently the long-term retention of $\mathrm{Gd}^{3+}$-based $\mathrm{CAs}$ in brains patients with normal renal function raised another possible safety issue. The safety concerns of $\mathrm{Gd}^{3+}$-based CAs have been based on the ligand structure of $\mathrm{Gd}^{3+}$-based CAs, and findings that $\mathrm{Gd}^{3+}$-based $\mathrm{CAs}$ with linear ligand structures showed much higher incidences of NSF and brain retention of CAs than $\mathrm{Gd}^{3+}$-based CAs with macrocyclic ligand structure. In the current study, we report the in vivo biodistribution profile of a new highly stable multifunctional $\mathrm{Gd}^{3+}$-based $\mathrm{CA}$, with macrocyclic ligand structure (HNP-2006). MR imaging using HNP-2006 demonstrated a significant contrast enhancement in many different organs. Furthermore, the contrast enhanced tumor imaging using HNP-2006 confirmed that this new macrocyclic CA can be used for detecting tumor in the central nervous system. Therefore, this new multifunctional HNP-2006 with macrocyclic ligand structure shows great promise for whole-body clinical application.

Keywords: Gadolinium contrast agent; Biodistribution profile; Macrocyclic structure; Safety

Small molecular weight gadolinium chelate is a positive contrast agent (CA) for magnetic resonance imaging (MRI), used to diagnose many tissue and vascular diseases that may not be detected in unenhanced MR images (1). However, over the last decade, safety issues have arisen regarding the use of gadolinium CAs, because some $\mathrm{Gd}^{3+}$ based CAs have been associated with nephrogenic systemic fibrosis (NSF), that can arise in patients with impaired renal function $(2,3)$. Furthermore, recent reports revealed another possible safety issue when long-term retention of $\mathrm{Gd}^{3+}$-based $\mathrm{CAs}$, was found in the brains of patients with normal renal function (4).

There is a consensus in the scientific literature that NSF and brain retention of $\mathrm{Gd}^{3+}$ based CAs are strongly associated with the ligand structure of $\mathrm{Gd}^{3+}$-based CAs. Several 
studies have shown that $\mathrm{Gd}^{3+}$-based CAs with linear ligand structures were associated with a much higher incidence for NSF and brain retention of CAs, than $\mathrm{Gd}^{3+}$-based CAs with macrocyclic ligand structures $(3,5)$. The underlying principle behind the difference between the two types of ligand structures is that the $\mathrm{Gd}^{3+}$-based CAs with linear ligand structure are less resistant to the release of free gadolinium than the $\mathrm{Gd}^{3+}$-based CAs with macrocyclic ligand structure (6). Due to safety concerns, among all commercialized $\mathrm{Gd}^{3+}$ based CAs with linear ligand structures, 4 extracellular fluid (ECF) CAs were recommended for withdrawal from the market in Europe (7). However, the liver-specific $\mathrm{Gd}^{3+}$-based CAs with linear ligand structures, were not recommended for market withdrawal because there are no functional $\mathrm{Gd}^{3+}$-based CAs with macrocyclic ligand structures.

Recently, we synthesized a highly stable multifunctional $\mathrm{Gd}^{3+}$-based CA with a macrocyclic ligand structure (HNP-2006) (8). In the current study, we report the in vivo biodistribution profile of this new multifunctional $\mathrm{Gd}^{3+}$-based CA. We also demonstrated that this new multifunctional $\mathrm{Gd}^{3+}$-based $\mathrm{CA}$, with macrocyclic ligand structure holds promise for several clinical imaging applications.

In vivo MRI biodistribution experiments were performed using a 1.5T MRI system (GE Healthcare, Milwaukee, WI, USA). The experimental protocol was approved by the animal research committee of Kyungpook National University (KNU). Homemade birdcage-type radiofrequency (RF) coil, which was a receiver-only type with an inner diameter of $50 \mathrm{~mm}$, was used for small animals. The imaging parameters for spin echo sequence were as follows: repetition time $(T R)=300 \mathrm{~ms}$; echo time $(T E)=13 \mathrm{~ms}$; field of view $(F O V)=8 \mathrm{~mm} ; 192 \times 128$ matrix size; $1.2 \mathrm{~mm}$ slice thickness; number of excitation (NEX) $=8$. Total scan time was 2 minutes, 37 seconds. The imaging parameters for spin echo sequence were as follows: $\mathrm{TR}=300 \mathrm{~ms} ; \mathrm{TE}=13 \mathrm{~ms}$; FOV $=8 \mathrm{~mm} ; 192 \times 160$ matrix size; $1.2 \mathrm{~mm}$ slice thickness; $\mathrm{NEX}=8$. Total scan time was 2 minutes, 37 seconds. Sixweek old male mice (weighing 25-27 g), obtained from the Institute of Cancer Research (ICR) were used. Animals were anesthetized by $1.5 \%$ isoflurane in oxygen. For the tumor model, the mice were placed in a stereotaxic apparatus. After scalp incision to expose bregma, the skull was drilled at an exact position of $1 \mathrm{~mm}$ posterior and $2 \mathrm{~mm}$ lateral from the bregma for stereotaxic injections. C6 glioma cells were prepared $\left(1.0 \times 10^{5}\right)$ in DMEM, and injected for 5 minutes at a rate of $1 \mu \mathrm{L} / \mathrm{min}$, and depth of $2 \mathrm{~mm}$ into the hole. The injection needle was kept in this position to prevent cell leaks for 5 minutes, then slowly removed from the brain. The MR study was performed 2 weeks after C6 glioma implantation. MR images were acquired before and after tail vein injection of multifunctional $\mathrm{Gd}^{3+}$ based CA with macrocyclic ligand structure (HNP-2006). The CA injection dose was $0.1 \mathrm{mmol} \mathrm{Gd} / \mathrm{kg}$ for MR images. Anatomical locations of liver, bile duct, heart, and kidney were identified in post-contrast enhanced MR images. For quantitative measurements, the signal intensities in specific regions of interest (ROI) were measured using Advantage Window software (GE Medical, USA). The contrast-to-noise ratio (CNR) was calculated using equation [1], where SNR is the signal-to-noise ratio.

$$
\mathrm{CNR}=\mathrm{SNR}_{\text {post }}=\mathrm{SNR}_{\text {pre }}
$$

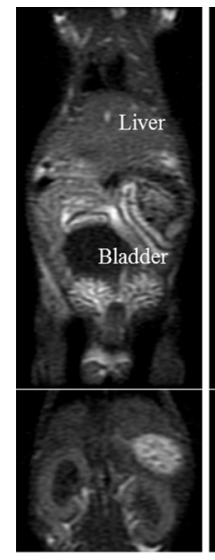

Pre

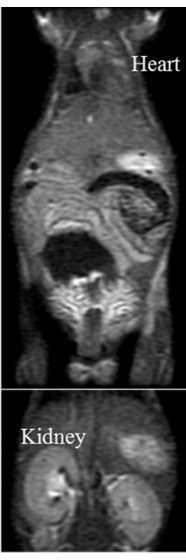

Post $5 \mathrm{~min}$

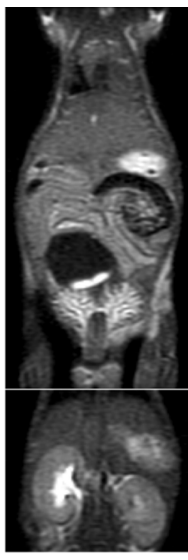

Post $10 \mathrm{~min}$

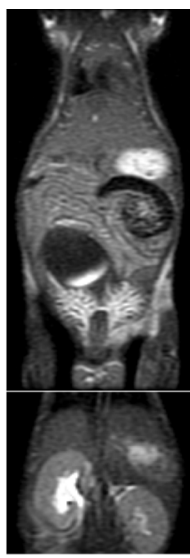

Post $30 \mathrm{~min}$

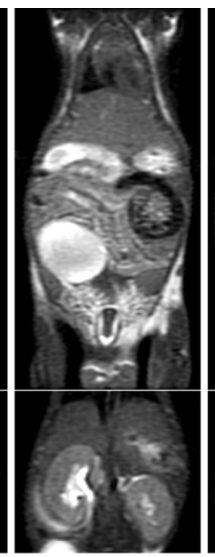

Post $1 \mathrm{~h}$

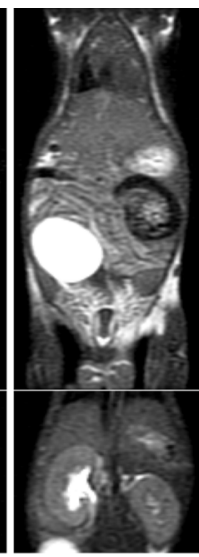

Post $1.5 \mathrm{~h}$

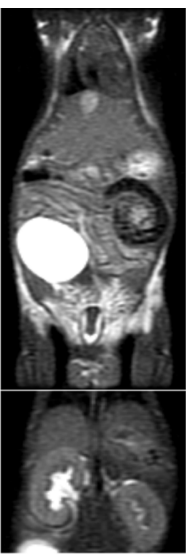

Post $2 \mathrm{~h}$

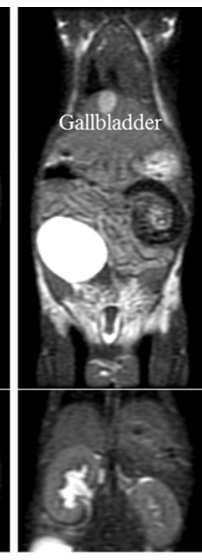

Post $2.5 \mathrm{~h}$

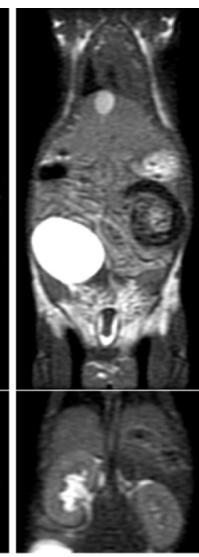

Post $3 \mathrm{~h}$

Fig. 1. Coronal T1-weighted images of 6-week-old male ICR mice before and after a highly stable multifunctional $\mathrm{Gd}^{3+}-$ based CA with macrocyclic ligand structure (HNP-2006) injection. 
Figure 1 shows the coronal T1-weighted images of 6-week-old male ICR mice before and after HNP-2006 injection. Figures 1 and 2 demonstrated that several organs showed significant contrast enhancements after CA injection. The strong heart enhancement demonstrated that HNP-2006 can be used for possible cardiovascular application. The most characteristic features of HNP2006 as a CA are that the liver and gallbladder showed significant signal enhancement. Contrast enhancement of the liver and gallbladder established HNP-2006 as the first $\mathrm{Gd}^{3+}$-based CAs with macrocyclic ligand structure, with possible hepatobiliary applications. Currently, two $\mathrm{Gd}^{3+}$-based CAs with linear ligand structures, Gd-BOPTA (Multihance) and Gd-EOB-DTPA (Primovist), were reported to show enhancement of the liver and gallbladder. The in vivo biodistribution patterns of Gd-BOPTA and Gd-EOBDTPA were thought to result from the fact that these agents exhibit lipophilic properties, and thus are excreted through the hepatocytes and biliary tract. In the hepatocyte, these agents are known to be excreted through organic anion-

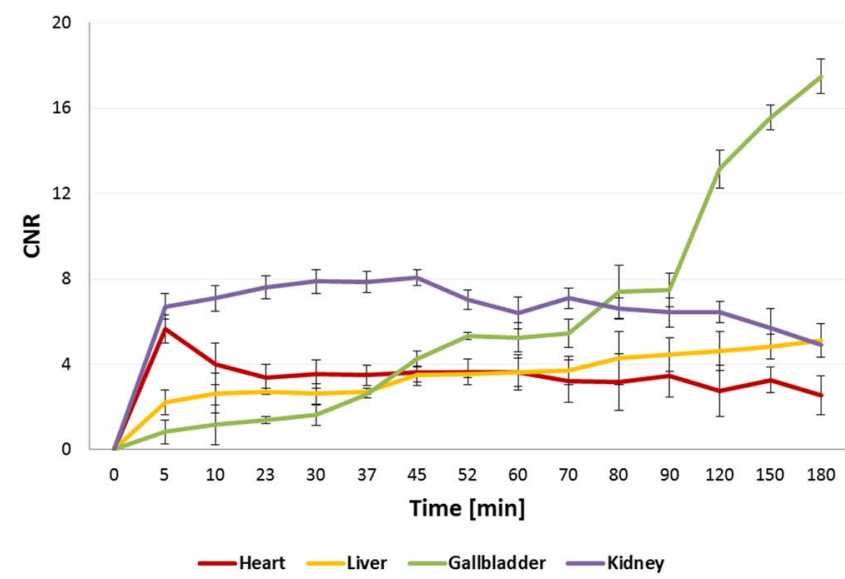

Fig. 2. Time-CNR curve of HNP-2006 in various organs. transporting polypeptide-mediated protein channels (9). Therefore, HNP-2006 seems to use a similar organic anion-transporting polypeptide-mediated protein channel for hepatocyte uptake. Furthermore, the strong contrast enhancement in kidney and bladder confirmed that HNP2006 was excreted by glomerular filtration, in addition to hepatobiliary excretion. The in vivo biodistribution pattern of HNP-2006 through kidney and bladder is very similar with that of commercialized macrocyclic $\mathrm{Gd}^{3+}$-based ECF agents, such as Gd-DOTA (Dotarem ${ }^{\circledR}$ ) and Gd-HP-D03A (Prohance ${ }^{\circledR}$ ). After injection, these CAs were excreted by the kidneys and administration of these CAs resulted in the same diagnostic imaging and in vivo biodistribution.

Figure 3 shows the coronal T1-weighted images of brain tumors in an animal model before and after HNP2006 injection. The tumor, which was not visible in the pre-contrast image, was clearly depicted in the postcontrast image. Figure 4 shows the significant difference in CNR between tumor and normal brain tissue. Time series data suggested that wash out patterns differentiated the

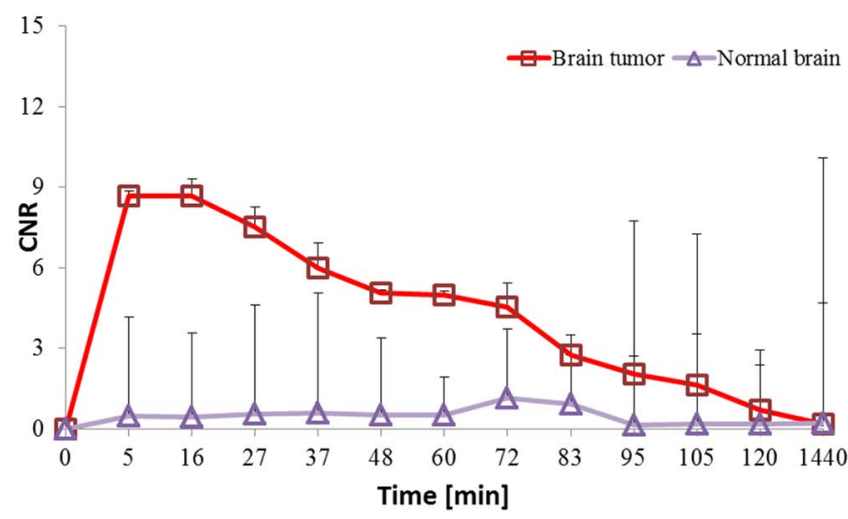

Fig. 4. Time-CNR curve of HNP-2006 in tumor and normal brain tissue.

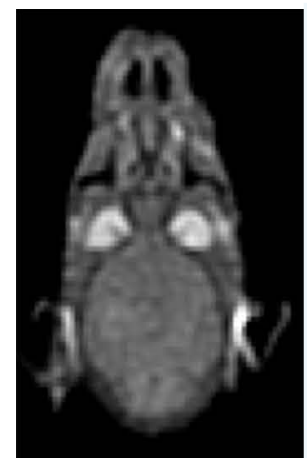

Pre

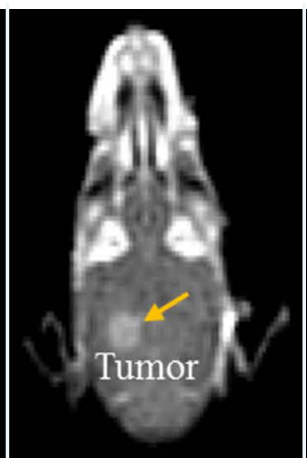

Post $5 \mathrm{~min}$

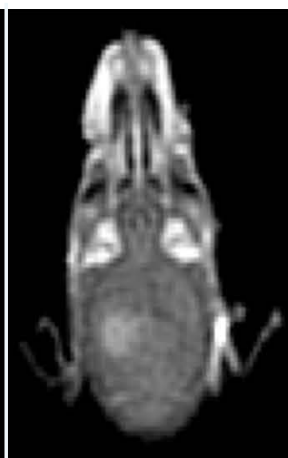

Post $30 \mathrm{~min}$

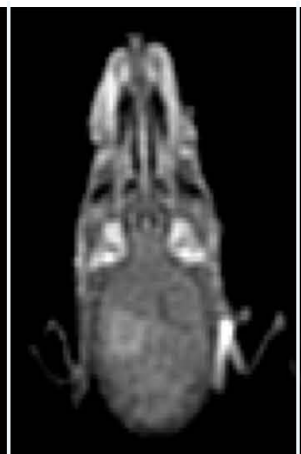

Post $1 \mathrm{~h}$

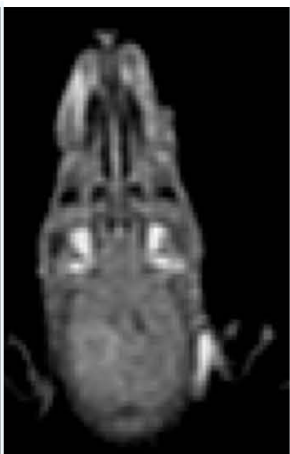

Post $2 \mathrm{~h}$

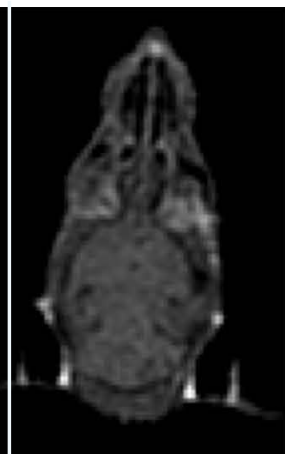

Post $24 \mathrm{~h}$

Fig. 3. Coronal T1-weighted images of brain tumor model before and after HNP-2006 injection. 
active tumor margin from the central necrosis-like region. Therefore, tumor images using HNP-2006 confirmed that HNP-2006 can effectively be used for enhanced imaging.

To our knowledge, HNP-2006 is the first macrocyclic $\mathrm{Gd}^{3+}$-based multifunctional CA. As mentioned above, several macrocyclic $\mathrm{Gd}^{3+}$-based ECF CAs have already been commercialized. However, the macrocyclic $\mathrm{Gd}^{3+}$-based functional CA is not commercially available. For example, the $\mathrm{Gd}^{3+}$-based liver specific CAs, Gd-BOPTA (Multihance) and Gd-EOB-DTPA (Primovist), have linear ligand structures, and thus showed relatively low kinetic stability compared with HNP-2006 (8). The low stability of the linear type liver-specific CAs may increase the chance of release of free $\mathrm{Gd}^{3+}$ ions, compared to the macrocyclic HNP-2006. This instability of linear type functional CAs may be implicated in the higher incidence of in vivo toxicities, such as NSF and brain accumulation, compared to HNP-2006 with macrocyclic ligand structure.

In conclusion, the in vivo biodistribution profile of a new multifunctional $\mathrm{Gd}^{3+}$-based CA (HNP-2006) demonstrated significant contrast enhancement in many different organs. Furthermore, contrast enhanced tumor imaging using HNP-2006 confirmed that this new macrocyclic CA can be used for detecting tumors in the central nervous system. Therefore, this new multifunctional $\mathrm{Gd}^{3+}$-based CAs with macrocyclic ligand structure, is a promising contrast agent for use in whole-body clinical applications.

\section{Notes}

The authors declare no competing financial interest.

\section{Acknowledgments}

This work was supported by the Industrial Strategic Technology Development Program (Grant number: 10078376) funded by the Ministry of Trade, Industry \& Energy (MOTIE, Korea).

\section{REFERENCES}

1. Geraldes $C F$, Laurent $\mathrm{S}$. Classification and basic properties of contrast agents for magnetic resonance imaging. Contrast Media Mol Imaging 2009;4:1-23

2. Grobner T. Gadolinium--a specific trigger for the development of nephrogenic fibrosing dermopathy and nephrogenic systemic fibrosis? Nephrol Dial Transplant 2006;21:1104-1108

3. Marckmann P, Skov L, Rossen K, et al. Nephrogenic systemic fibrosis: suspected causative role of gadodiamide used for contrast-enhanced magnetic resonance imaging. J Am Soc Nephrol 2006;17:2359-2362

4. Kanda T, Fukusato T, Matsuda $M$, et al. Gadolinium-based contrast agent accumulates in the brain even in subjects without severe renal dysfunction: evaluation of autopsy brain specimens with inductively coupled plasma mass spectroscopy. Radiology 2015;276:228-232

5. Broome DR. Nephrogenic systemic fibrosis associated with gadolinium based contrast agents: a summary of the medical literature reporting. Eur J Radiol 2008;66:230-234

6. Kim HK, Lee GH, Chang Y. Gadolinium as an MRI contrast agent. Future Med Chem 2018;10:639-661

7. European Medicines Agency. PRAC concludes assessment of gadolinium agents used in body scans and recommends regulatory actions, including suspension for some marketing authorisations. 2017

8. Gu S, Kim HK, Lee GH, Kang BS, Chang Y, Kim TJ. Gd-complexes of 1,4,7,10-tetraazacyclododecane$\mathrm{N}, \mathrm{N}, \mathrm{N} ", \mathrm{~N} " '-1,4,7,10$-tetraacetic acid (DOTA) conjugates of tranexamates as a new class of blood-pool magnetic resonance imaging contrast agents. J Med Chem 2011;54:143-152

9. Narita $M$, Hatano $E$, Arizono $S$, et al. Expression of OATP1B3 determines uptake of $\mathrm{Gd}$-EOB-DTPA in hepatocellular carcinoma. J Gastroenterol 2009;44:793-798 